\title{
EL PROCESO DE FORMACIÓN INVESTIGATIVA DE LOS DOCENTES
}

PhD. Belinda Lema Cachinell Instituto Tecnológico de Formación martalema@formacion.edu.ec
Lic. Dennise Díaz Saavedra Instituto Tecnológico de Formación dennise.diaz@formacion.edu.ec
Ing. Lissette Vacacela Conforme Instituto Tecnológico de Formación lissette.vacacela@formacion.edu.ec
Palabras claves: investigación, proceso de formación docente.

Keywords: research, teacher training process.
Recibido: 06 de febrero de 2017

Aceptado: 24 de febrero de 2017

\section{RESUMEN}

El presente artículo realiza un análisis de la importancia de la formación del profesional en nuestros días como elemento imprescindible para fomentar hábitos de trabajo independiente y habilidades para su desarrollo como futuro investigador. De ahí que promover tareas en este sentido en estudiantes de la enseñanza superior presupone que la formación de un pensamiento creador, innovador, que posibilita la independencia intelectual, el espíritu investigativo, la solución de las tareas prácticas e intelectuales, lo que determina el progreso científico social futuro. Por lo tanto, para lograr la calidad de la educación superior, necesaria en estos tiempos, se requiere de un docente con un alto nivel de competencias, como premisa para comprender la realidad educativa desde su orientación y problematización y en consecuencia, tomar decisiones y actuar en su transformación en beneficio de toda la sociedad.

\section{ABSTRACT}

The present article analyzes the importance of the professional formation process nowadays as fundamental element to enhance research skills in professors. Thus, promoting tasks in students of higher education means the formation of a creative innovative thought that allow intellectual independence, research spirit, solution of practical e intellectual tasks, which in long term determines the future social scientific development. Therefore, in order to achieve quality in the higher education, necessary nowadays, a teacher with a high level of competences is needed as a premise to understand the educational reality from its orientation and as a result, make decisions and acting in its transformation benefiting the society. 


\section{INTRODUCCIÓN}

La presente investigación aborda el proceso formativo del hombre el cual se mueven en dimensiones, que se relacionan dialécticamente y se diferencian, ante todo, en su intención, en lo que persiguen: el educativo - la formación del hombre para la vida-, el instructivo - la formación del hombre como trabajador para aportar a la sociedad y al desarrollo humano, el desarrollador - la formación de sus potencialidades funcionales o facultades.

En sentido general, y de manera no contradictoria a lo anterior, es entendida la formación en el orden pedagógico, psicológico y personológico, a lo cual han hecho referencia pedagogos como Álvarez de Zayas (1999), Baxter (2002) y Chávez (2012), entre otros. A partir de analizar sus criterios se comprende la formación como la trascendencia personalizada o colectiva de la educación, que se evidencia en los logros del desarrollo humano, el crecimiento personal del individuo o del grupo en conformidad con los fines sociales y direcciones hacia la cual se orienta, en cuya esencia está el aprendizaje valioso; de ahí que sea una consecuencia de este. (Flores, 2005).

La formación se expresa en los conocimientos, habilidades, hábitos, valores y actitudes, individual y de grupo. Es un proceso complejo, contradictorio, de configuración personal, de relación de lo social y lo individual, del medio más cercano y las experiencias e historia personal.

En el profesional se requiere de la continuidad del proceso de formación, comprendida como la formación profesional que constituyen las acciones formativas que capacitan para el desempeño cualificado de las diversas profesiones, favoreciendo el acceso al empleo y participación activa en la vida social, cultural y económica.

\section{DESARROLLO}

Los debates en torno a la formación investigativa del docente, como parte de su formación permanente, han tenido como base, la polémica de la relación entre docencia e investigación, teoría y práctica y la visión del maestro investigador y el docente universitario que actúa como docente e investigador, a la vez.

La expresión "maestro investigador" se vincula a Stenhouse (1985) relacionada con un enfoque curricular que hace énfasis en la relevancia de los contenidos a procesos de investigación (citado por Díaz, 2010)

Según Stenhouse (1985) el maestro investigador reivindica el derecho y la obligación profesional a ser él mismo constructor de su propio conocimiento y a tener un desarrollo profesional autónomo mediante la reflexión y el estudio de los trabajos de otros profesionales y la comparación de sus ideas mediante la investigación en el aula"

La investigación en el aula y de aula son estrategias de enseñanza participativas (Stenhouse, 2004), con ellas es posible afirmar una pedagogía de la investigación como proceso intencionado de formación en el nivel de pregrado en las universidades, asumiendo que el mayor peso de su realización descansa en el docente que debe desarrollar un recorrido científico a la par de un proceso de educación, haciendo explicita la intención de construcción de conocimientos y el 
trabajo de reflexión sobre el proceso con los estudiantes desde otro tipo de contextos de formación.

En consonancia con esta postura de base, otros autores, asumen posiciones similares, así por ejemplo Lutgarda et al (1999), consideran al maestro investigador como a aquel que sus conocimientos de la práctica educativa le permiten resolver los problemas de su escuela por la vía de la investigación.

Por su parte, Chirino (1997) considera que el maestro investigador es aquel que sin abandonar el aula de clase, cuya vivencia es irrepetible, es capaz de buscar alternativas de solución a los problemas de su quehacer profesional por la vía de la ciencia.

García (1999) concibe al maestro investigador como "aquel que posee la base del conocimiento del método científico, lo aplica en su labor pedagógica cotidiana y logra que el alumno se apropie de ella al instrumentar el proceso de enseñanza aprendizaje sobre bases más científicas" (citado por López L. y Pérez C. 1999).

El análisis crítico de tales concepciones, permiten señalar que se comprende el papel del método científico como la herramienta por excelencia para transformar la práctica pedagógica y, favorecer con ello, la apropiación de dicha herramienta por parte de los estudiantes en el aprender a aprender; sin embargo, no se advierte explícitamente cómo puede transcurrir desde la relación dialéctica teoría- práctica, el proceso paralelo o dual de aprender y enseñar a investigar.

La formación investigativa del docente, enfocada desde la perspectiva de maestro investigador, tiene en la investigación formativa, una vía esencial para alcanzar un desempeño investigativo favorable, aunque no suficiente para ser competente, dado las exigencias de investigar como función crucial en la educación superior.

La investigación formativa: "es un tipo de investigación que se hace entre estudiantes y docentes en el proceso de desarrollo del currículo de un programa y que es propio de la dinámica de la relación con el conocimiento que debe existir en todos los procesos académicos tanto en el aprendizaje, por parte de los alumnos, como en la renovación de la práctica pedagógica por parte de los docentes" (Restrepo, 2002).

Por su parte, Parra (2009) expresa que la investigación formativa exige al profesor universitario adoptar una postura diferente frente al objeto de enseñanza y frente a los estudiantes; en la primera resaltar el carácter complejo, dinámico y progresivo del conocimiento y en la segunda, reconocer y aceptar las potencialidades de los estudiantes para asumir la responsabilidad de ser protagonistas de su aprendizaje.

El mismo autor, significa que la investigación formativa se refiere a la investigación como herramienta del proceso enseñanza - aprendizaje, es decir, su finalidad es, difundir información existente y favorecer que el estudiante la incorpore como conocimiento (aprendizaje). La investigación formativa también puede denominarse a la enseñanza a través de la investigación, o enseñar usando el método de investigación. La investigación formativa tiene dos características adicionales fundamentales: es una investigación dirigida y orientada por un profesor, como parte de su función docente y los agentes investigadores no son profesionales de la investigación, sino sujetos en formación. 
Por otra parte, la calidad de la formación científica descansa en la índole misma de la docencia, en la construcción en el aula de un ambiente interactivo que propicie un proceso compartido de construcción del conocimiento, pero con un alto grado de dirección y compromiso docente con unas condiciones de apoyo e infraestructura adecuadas a los propósitos de la formación, incluyendo el ámbito de la relación ciencia, tecnología y sociedad. (Patiño, 2007).

Tales concepciones que resaltan el papel de los actores fundamentales del proceso de enseñanza- aprendizaje, en el uso del método científico como herramienta para el aprendizaje de todos los sujetos. Sin embargo, no se advierte explícitamente, cómo favorecer dicha formación desde una mirada de competencias, en la que, de manera simultánea, el docente aprenda a investigar y enseñe a investigar a los estudiantes, sustentada en la motivación y el compromiso con el cambio y la mejora continua de la calidad educacional.

En esta primera arista o dimensión de la reflexión teórica y práctica en torno a la formación investigativa del docente, se debe sistematizar desde la práctica pedagógica del docente, el método de aprendizaje basado en problemas (ABP), que sigue la lógica de la investigación científica. Se coincide con Parra (2009) cuando, al referirse a este método, expresa: "esta estrategia que permite que el estudiante aprenda a aprender, aprenda a pensar de manera crítica y analítica, y a buscar, encontrar y utilizar los recursos apropiados para aprender".

De igual modo, a los fines de significar el papel de ciertas herramientas para conducir el aprendizaje orientado hacia la investigación, se consideran positivas algunas de las regularidades metodológicas en la formación de profesionales técnicos, aportadas por Cortijo (1996), en Didáctica de las Ramas Técnicas, entre las que figuran: método de trabajo tecnológico- método de enseñanza, aprendizaje por problemas- dominio de lo esencial del contenido de las ciencias, realización de proyectos- ejecución de tareas específicas, aprendizaje en el proceso profesionalaprendizaje en condiciones simuladas y experiencia de aprendizaje- competencia profesionales.

La segunda visión que se connota en la formación investigativa del docente, es la relativa a la formación en investigación o formación investigativa, la que está en consonancia con uno de los procesos sustantivos de la educación superior, que es la investigación científica, concretada en la actividad de ciencia e innovación tecnológica, lo cual exige, contar con docentes que deben cumplir las funciones de docente e investigador.

Al respecto, Restrepo (2002) expresó que la investigación universitaria es un proceso de búsqueda de nuevo conocimiento, proceso caracterizado por la creatividad del acto, por la innovación de ideas, por los métodos rigurosos utilizados, por validación y juicio crítico de pares. A la investigación está unida íntimamente la creatividad ya que en buena medida los resultados de la investigación son también creación de conocimiento o de tecnología y la investigación científica debe operar en la universidad no sólo en el ámbito de las disciplinas o ciencias básicas, sean éstas naturales, formales o sociales, sino también en el ámbito de las profesiones 0 carreras.

Por su parte, Guerrero (2007) define formación para la investigación: "al conjunto de acciones orientadas a favorecer la apropiación y desarrollo de los conocimientos, habilidades y actitudes necesarios para que estudiantes y profesores puedan desempeñar con éxito actividades productivas asociadas a la investigación 
científica, el desarrollo tecnológico y la innovación, ya sea en el sector académico o en el productivo".

Por su parte, Medina y Barquero (2012), expresan que, "se aprende a investigar, investigando, es decir, la forma más efectiva de aprender a investigar, de adquirir los conocimientos, habilidades, valores y actitudes que precisa el quehacer investigativo, es, realizando investigación.

Se coincide, en general, con tales posturas, ya que se necesita investigar, no solo en el ámbito de las disciplinas en el proceso de enseñanza aprendizaje y la labor educativa que trasciende a todos los escenarios de la formación del profesional, sino también, en el ámbito de las profesiones o carreras, que corresponde a los procesos productivos y de servicios para los que se forman los alumnos, y por supuesto, imprimir creatividad a lo que se hace, así como jerarquizar la necesidad de la formación investigativa en estudiantes y profesores.

Como síntesis de las posiciones teóricas de los autores a las que se ha hecho alusión, se puede expresar que, de una manera u otra, advierten acerca de la necesidad de que el docente universitario posea una adecuada formación investigativa, que le permita mejorar su práctica pedagógica y, que ello, se exprese al mismo tiempo, en la formación investigativa del estudiante, para poder enfrentar los problemas en el ejercicio de la profesión.

Por otro lado, revelan la necesidad de que el docente, sea capaz de producir conocimientos científicos, para resolver problemas de la práctica y de la teoría pedagógica, así como de las ciencias y procesos técnicos y tecnológicos que sustentan su labor.

Tal intención, se complementa, en cierta medida con el planteamiento de Begoña (2008), al concebir al profesor universitario como: "aquel docente e investigador a la vez, que reflexiona sobre y desde su práctica educativa, lo que coadyuva a mejorarla en función de las necesidades contextuales donde se desenvuelve" .En esta definición, se destacan dos aspectos que son esenciales y que diferencian al profesor universitario del resto de los niveles de enseñanza precedentes: a la vez que imparte clases, investiga.

El análisis y valoración crítica realizada hasta aquí, permite advertir un predominio de concepciones de formación investigativa de docentes, que jerarquizan la visión de maestro investigador y formación del docente para la investigación, sustentado en el debate de las relaciones, entre docencia e investigación, así como entre teoría y práctica, esencialmente.

Sin embargo, se necesita repensar la formación investigativa en el docente, desde una mirada de competencia investigativa, dado que, en la actualidad, el componente investigativo, se erige como un parámetro de calidad de la educación superior, obligatoria en los procesos de acreditación de los programas académicos. Para lo cual se significa, no solo investigar, sino utilizar la investigación debidamente en el proceso de formación, instando a la comunidad educativa a valerse de ella, para solucionar problemas, fundamentalmente de carácter pedagógico. 
En los últimos años, se han realizado investigaciones que apuntan a concepciones y prácticas a favor de la formación de la competencia investigativa del docente, con énfasis en los docentes de la educación superior, que incluye, a los de los Institutos Superiores de Tecnología; dichas concepciones, responden a ciertos enfoques de competencias que la sustentan, ya sea conductista, funcionalista, constructivista 0 enfoque holístico o complejo; este último, defendido por Tobón, (2007).

A los fines de la presente investigación, las definiciones de competencias y de competencia profesional, que se aproximan a la intencionalidad que se defiende, en cuanto al modo, en que debe producirse la formación de la competencia investigativa del docente, y que, al mismo tiempo, permitirá sistematizar la teoría existente, como base aportar una definición propia, son las aportadas por: González (2002); Forgas, (2003), Castellanos et. al., (2003) y Tobón, (2007).

Se asume la definición de competencia profesional aportada por Forgas, (2003), que se refuerza con los términos comprensión y emprendimiento, mejoramiento continuo y compromiso ético.

Lo anterior, facilita la comprensión y asunción de posiciones críticas en torno a la noción de competencia investigativa, como resultado de un proceso de formación investigativa del docente, que tiene lugar en y desde su actuación pedagógica profesional, en un contexto histórico dado.

Maldonado et al. (2007) sostuvieron que "la competencia investigativa propende por la aplicación de los conocimientos, enfatizando en las diferentes esferas involucradas en la actividad investigativa entre las que se destacan las dimensiones epistemológica, metodológica, técnica y social".

Se comparte el criterio, de que esta concepción trae consigo que se limita la competencia a la aplicación de conocimientos asociados a la investigación científica, sin tener en cuenta que el saber, no implica el saber ser, ni saber hacer.

En tal sentido la revisión de literatura ofrece una definición presentada por Castellanos (2001), en la que especifica a la competencia para la investigación educativa como aquella que permite a los profesionales de la educación como sujetos cognoscentes, la construcción del conocimiento científico acerca del proceso pedagógico en general y del proceso de enseñanza - aprendizaje en particular, con el propósito de solucionar eficientemente los problemas en el contexto de la comunidad educativa escolar.

En el plano psicológico, dado las particularidades del docente joven /adulto, poseedor de experiencias y motivaciones, es conveniente que, a la hora de implicarlo intencionalmente, en un proceso de formación investigativa permanente, teniendo como base y aspecto primordial, la autorreflexión y reflexión crítica de su propia_práctica pedagógica, sea aconsejable, considerar algunos aspectos de interés en torno al funcionamiento de la personalidad desde una perspectiva integral.

El primer aspecto a considerar, tiene que ver con la esfera inductora o motivacional afectiva; es decir, lo relativo, al por qué y al para qué de la actuación investigativa del docente. En esa dirección, es preciso identificar los motivos e intereses que sustentan su posible actuación investigativa: interés cognoscitivo, desarrollar una docencia basada en la investigación, saber caracterizar integralmente a sus 
estudiantes y dar seguimiento al diagnóstico pedagógico integral, producir conocimientos científicos, tener un status de reconocimiento profesional y social, entre otros; de modo, similar, hay que hacerlo con los estudiantes.

Por otro lado, en la esfera ejecutora o cognitiva - instrumental, es preciso constatar por diferentes vías, los conocimientos, habilidades, valores de tipos, profesionales, morales e intelectuales, que deben tipificar su competencia investigativa individual, como parte de su desempeño profesional y, su correspondencia con exigencias institucionales y sociales.

Por último, es imprescindible, considerar la categoría autorregulación y la metacognición y, sus implicaciones en la actuación y formación permanente de los docentes.

Uno de los aspectos cruciales que garantiza el éxito del proceso de enseñanzaaprendizaje viene dado por el "aprender a aprender", que debe ser internalizado y adoptado por la persona en su papel de aprendiz; en este proceso, se deberá considerar la metacognición y autorregulación del aprendizaje, como parte de las estrategias que son imprescindibles aplicar para el éxito de su formación integral, y de manera particular, la formación investigativa.

Cada vez más, se justifica la necesidad de aprender a conocer, aprender a actuar, aprender a vivir juntos y aprender a ser, "pilares de la educación" reconocidos en el mundo y que preconiza la UNESCO sobre los que debe sustentarse la educación de las nuevas generaciones de niños, adolescentes y jóvenes.

\section{CONCLUSIONES}

Se puede valorar a manera de conclusión que el que es necesario replantear y contextualizar la concepción de competencia investigativa para el docente de los institutos tecnológicos, como parte de su profesionalización docente, ya que, en general, las competencias deben preparar al hombre para la vida, mucho más allá, de dar respuestas a las demandas puntuales de un puesto de trabajo o área ocupacional.

Por lo tanto, se valora en la presente investigación que la competencia investigativa del docente debe ser producto de una dinámica de aprendizaje continuo, que interrelacione cada vez más, a planos superiores, la práctica reflexiva del docente, su capacitación continua y la experimentación de procesos formativos para la transformación de la práctica, sustentados en la motivación, el compromiso con la mejora continua y la autoformación permanente. 


\section{REFERENCIAS BIBLIOGRÁFICAS}

Álvarez, C. (1999). La Escuela en la Vida. La Habana: Editorial Pueblo y Educación

Báxter, E. (2002). ¿Cuándo y cómo educar en valores? Instituto Central de Ciencias Pedagógicas-ICCP: Ministerio de Educación- MINED. La Habana, Cuba: Recuperado de https://issuu.com/hansmejiaguerrero/docs/ibro.cu_ndo_y_c_mo_educar _en_val/1

Castellanos, D. (2001). Hacia una concepción del aprendizaje desarrollador. La Habana, Cuba: Revista Varona. Colección Proyectos.

Castellanos, D. y otros (2001). Para promover un aprendizaje desarrollador. Colección Proyectos. Material digitalizado. La Habana: Centro de Estudios Educacionales del I.S.P. "Enrique José Varona.

Chávez, J. (2012). Filosofía de la educación para maestros. Cuba. Recuperado de www.cubaeduca.cu/medias/pdf/2778.pdf

Chirino, V. (1997). ¿Cómo formar maestros investigadores? Curso 59. Pedagogía '97, La Habana: PALCOGRAF.

Cortijo, R. (1996). Didácticas de las Ramas Técnicas: una alternativa para su desarrollo. Tesis para optar por el título de Máster en Ciencias de la Educación. La Habana: CEPROF, ISPETP.

Díaz, A. (2010). Los profesores ante las innovaciones curriculares. Revista Iberoamericana de Educación Superior (RIES), México, IISUE-UNAM/ Universia. 1, (1), pp. 37-57.

Flores, R. (2005). Pedagogía del conocimiento. Editorial McGraw Hill. Recuperado de http://www.monografias.com/trabajos72/modelos-pedagogicoscontemporáneos/modelos-pedagogicos-contemporaneos2

Forgas, J. (2003). Diseño Curricular por Competencias. Folleto básico del curso. Quito: Recuperado de http// www.campus-oei.org/eduytrabajo

. (2003). La Formación por Competencias del Técnico de Nivel Medio en Mecánica de Taller en Cuba: Una alternativa del diseño curricular. La Habana, Cuba: PEDAGOGÍA

(2003). La Formación Profesional Basada en Competencias Profesionales: una alternativa para el diseño curricular en el nuevo milenio. p.17 - p.19. - En Revista electrónica "Maestro y Sociedad" Santiago de Cuba, No. 4, Cuba. 
(2003). Modelo curricular para la formación del técnico de nivel medio basado en competencias profesionales. Tesis en opción al grado científico de Doctor en Ciencias Pedagógicas. Santiago de Cuba, Cuba.

García, I. (1999). Maestro investigador: Inteligencia, talento y creatividad para aprender y enseñar. Curso 42, Pedagogía '99. La Habana: PALCOGRAF.

Guerrero, M. (2007). Formación para la investigación en el contexto universitario. Bogotá: Editorial Universidad Católica de Colombia.

González. V. (2002). ¿Qué significa ser un profesional competente? Reflexiones desde una perspectiva psicológica. Revista Cubana de Educación Superior, Vol. XII, No. 1, 2002, pp. 45-53.

(2002). La orientación profesional en la educación superior. Una alternativa teórico-metodológica para la formación de profesionales competentes. Ponencia. 3era Convención Internacional de Educación Superior. Monografía. CEPES Universidad de la Habana. Ciudad Habana. Cuba

López, L. (1999). El desarrollo de las habilidades de investigación en la formación inicial del profesorado. La Habana: MINED

López, L. y Pérez C. (1999). Maestro investigador: ¿Cómo lograrlo? Curso 26, Pedagogía '99, Ed. Palcograf, La Habana.

Maldonado, L. et al (2007). abril-junio. Visibilidad y formación en investigación. Estrategias para el desarrollo de competencias investigativas. Studiositas, 2(2), 43-56. Recuperado http://dialnet.unirioja.es/servlet/articulo?codigo=2719652

Medina, M. y Barquero, J. (2012). Mi última investigación científica: 20 competencias profesionales para la práctica docente. México: Editorial Trillas.

Parra, C. (2009). Apuntes sobre la investigación formativa. Indexada en: ISI - Scielo Citation Index, Scielo Redalyc, http:/educacionyeducadores.unisabana.edu.co/

Patiño, L. (2007). La docencia universitaria. Elementos para su práctica. Ibagué: Universidad de Ibagué

Prieto, M. (2004). La construcción de la identidad profesional del docente. Revista enfoque: un desafío permanente, en Fundamentos sociales de la educación e investigación cualitativa, Universidad católica de Valparaíso, Chile.

Restrepo, B. (2002). Una variante pedagógica de la investigación-acción educativa. Colombia. Recuperado de rieoei.org/deloslectores/370Restrepo.PDF 
Restrepo, B. (2002). Conceptos y aplicaciones de la investigación formativa, y criterios para evaluar la investigación científica en sentido estricto. Bogotá: CNA.

Rosovsky, H. (2010) La universidad. Cátedra UNESCO-UNU "Historia y Futuro de la Universidad" de la Universidad de Palermo: Argentina. ISBN 978- 9871716-02-9 302 pp. RAES. Revista Argentina de Educación Superior.

Stenhouse, L. (1985). El profesor como investigador Investigación y desarrollo del curriculum. Madrid, España: Morata. pp. 194 - 221.

. (2004). La investigación como base de la enseñanza. Selección de textos por J. Rudducky David Hopkins, 5 edición. Madrid: Morata.

Tobón, S. (2007). Gestión curricular y ciclos propedéuticos. Bogotá: ECOE. Tobón, S., García-Fraile, J.A., y otros. (2006). Competencias, calidad y educación superior. Bogotá: Magisterio 